\title{
PREVALÊNCIA DOS FATORES DE RISCO INTRÍNSECOS AO PACIENTE E O DESFECHO QUEDA NA CLÍNICA CIRÚRGICA*
}

\author{
Cristiane Ferraz da Silva ${ }^{1}$, Suelen Reiniack ${ }^{2}$, Bruna de Melo Souza ${ }^{3}$, Karinne Cristinne da Silva Cunha ${ }^{4}$
}

\begin{abstract}
RESUMO: Os objetivos do estudo foram identificar a prevalência dos fatores de risco de queda intrínsecos durante a internação do paciente e buscar os registros de queda de paciente no período de janeiro de 2015 em uma unidade de internação cirúrgica. Os participantes foram os pacientes da enfermaria cirúrgica de um hospital no Rio de Janeiro. A pesquisa foi realizada de janeiro a março de 2015. Considerou-se a presença ou ausência dos fatores de risco intrínsecos para queda. Foram registrados cinco $(5,1 \%)$ casos de queda do total de 98 participantes. Associação dos fatores de risco no paciente que sofreu queda mostrou que $100 \%$ (5) dos pacientes tinham o fator de risco comprometimento da visão, 80\% (4) apresentavam idade maior que 65 anos e anemia. O foco principal é reduzir os casos de queda no cenário de estudo desta pesquisa. Portanto, pesquisa futura pretende aprofundar o assunto entorno do desenvolvimento de tecnologia educacional para reduzir a incidência de queda no cenário de estudo.
\end{abstract}

DESCRITORES: Acidentes por quedas; Segurança do paciente; Fatores de risco; Medidas de segurança; Enfermagem.

\section{PREVALENCE OF INTRINSIC RISK FACTORS FOR PATIENTS IN FALL OUTCOMES IN A SURGICAL CLINIC}

ABSTRACT: This study aimed to identify the prevalence of intrinsic fall risk factors during a patient's hospitalization. It also involved a search for records of patients' falls in January 2015 in a surgical hospitalization unit. Research was conducted from January to March 2015. Presence and absence of risk factors intrinsic to falling were considered. Five cases (5.1\%) of falling were registered in 98 participants. Association of risk factors in patients that had fallen showed that $100 \%$ (5) of patients had risk factor of impaired vision, $80 \%$ (4) were more than 65 years old and had anemia. The principal focus was to reduce fall occurrences in the setting of this study. Future research intends to delve deeper on the subject of educational technology development in order to reduce the incidence of falling in the studied setting.

DESCRIPTORS: Accidental falls; Patient safety; Risk factors; Security measures; Nursing.

\section{PREVALENCIA DE LOS FACTORES DE RIESGO INTRÍNSECOS AL PACIENTE ASOCIADOS A CAÍDA EN LA CLÍNICA QUIRÚRGICA}

RESUMEN: Fueron objetivos del estudio identificar la predominancia de los factores de riesgo de caída intrínsecos durante la internación del paciente, así como buscar los registros de caída de paciente en el periodo de enero de 2015 en una unidad de internación quirúrgica. Los participantes fueron los pacientes de la enfermería quirúrgica de un hospital en Rio de Janeiro. La investigación fue realizada de enero a marzo de 2015. Fue considerada la presencia o ausencia de los factores de riesgo intrínsecos para caída. Se registraron cinco $(5,1 \%)$ casos de caída del total de 98 participantes. La asociación de los factores de riesgo del paciente que sufrió caída evidenció que $100 \%$ (5) de estos presentaban el factor de riesgo comprometimiento de la visión, 80\% (4) tenían edad mayor que 65 años y anemia. El foco principal es reducir los casos de caída en el escenario de estudio de esta investigación. Por lo tanto, una investigación futura puede profundizar el asunto del desarrollo de tecnología educacional para reducir la incidencia de caída en el escenario de estudio.

DESCRIPTORES: Accidentes por caídas; Seguridad del paciente; Factores de riesgo; Medidas de seguridad; Enfermería.

\footnotetext{
*Artigo extraído da dissertação intitulada: "Tecnologia educacional para prevenção de queda do paciente cirúrgico baseada na análise dos fatores de risco". Universidade Federal do Estado do Rio de Janeiro, 2016.

${ }^{1}$ Enfermeira. Mestranda do Programa de Pós-graduação em Saúde e Tecnologia no Espaço Hospitalar da Universidade Federal do Estado do Rio de Janeiro. Rio de Janeiro, RJ, Brasil.

${ }^{2}$ Enfermeira. Residente em Clínica Médica e Cirúrgica pela Universidade Federal do Estado do Rio de Janeiro. Rio de Janeiro, RJ, Brasil.

${ }^{3}$ Discente em Enfermagem. Universidade Federal do Estado do Rio de Janeiro. Rio de Janeiro, RJ, Brasil.

${ }^{4}$ Enfermeira. Doutora em Neuroimunologia. Docente da Universidade Federal do Estado do Rio de Janeiro. Rio de Janeiro, RJ, Brasil.
} 


\section{- INTRODUÇÃO}

A queda é descrita como sendo um deslocamento não intencional do corpo para um nível inferior à posição inicial, provocado por circunstâncias multifatoriais, resultando ou não em dano(1).

O Ministério da Saúde, juntamente com a ANVISA e a Fiocruz, divulgou no mês de maio de 2013 o Protocolo de Prevenção de Quedas, integrante do Programa Nacional de Segurança do Paciente ${ }^{(1)}$.

O Protocolo de Prevenção de Quedas deverá ser aplicado ao hospital, portanto incluirá todos os pacientes que recebem cuidado neste serviço, du rante o tempo de permanência em todos os ambientes do hospital. O protocolo publicado define como intervenção importante na admissão do paciente na instituição hospitalar a avaliação quanto ao risco de queda utilizando-se uma escala adequada ao perfil do paciente e a determinação dos fatores predisponentes específicos que aumentam o risco de queda. Essa avaliação deverá ser repetida diariamente até que o paciente receba alta hospitalar ${ }^{(1)}$.

A queda tem incidência de $12,4 \%$ em ambientes cirúrgicos, sendo considerado o evento adverso mais comum entre pacientes hospitalizados. Mas poderá ser mitigada por meio de estratégias de melhoria na estrutura do ambiente hospitalar e através de programa de prevenção ${ }^{(2)}$.

A mensuração do nível de risco do paciente sofrer queda pelo profissional de saúde é necessária para a promoção de intervenções preventivas, portanto é imprescindível que tais fatores de risco de queda sejam identificados com o intuito de evitar esse agravo à saúde ${ }^{(3)}$.

Os fatores de risco ambientais, como a disposição dos móveis, o tipo de superfície do chão, a presença de barras de segurança e os fatores de risco intrínsecos ao paciente que dizem respeito à sua marcha e locomoção relacionam-se e em algumas situações são interdependentes. Além disto, condições do pré-operatório e pós-operatório interferem e modificam o risco para queda.

Neste contexto, pensou-se na queda do paciente na enfermaria de clínica cirúrgica e nos aspectos multifatoriais que envolvem a sua ocorrência. Sendo assim, a identificação dos fatores de risco, suas prevalências e causas relacionais são fundamentais para a manutenção da segurança do paciente.

Justifica-se a escolha da temática porque a queda do paciente no ambiente hospitalar poderá causar desconforto e lesões de gravidade variável ao paciente, podendo levá-lo ao óbito ou agravar seu quadro clínico, dependendo da extensão do problema ocasionado. Além da ocorrência da queda contribuir de forma negativa para a imagem da instituição.

A análise de incidentes relacionados à segurança do paciente deverá ser feita no momento em que há perda dos níveis de qualidade na instituição. Neste intuito, ações deverão ser tomadas a fim de corrigir os erros para a melhoria do processo de trabalho(4).

Os objetivos deste estudo foram: identificar a prevalência dos fatores de risco de queda intrínsecos durante a internação do paciente e buscar os registros de queda de paciente no período de janeiro de 2015 em uma unidade de internação cirúrgica.

\section{MÉTODO}

Trata-se de um estudo epidemiológico de corte transversal. Os participantes foram os pacientes internados na enfermaria da clínica cirúrgica de um hospital no município do Rio de Janeiro. Todos os pacientes internados no mês de janeiro de 2015 entraram na amostra. Ao total foram entrevistados 99 participantes, apenas um foi excluído.

Os critérios de seleção da amostra foram: os pacientes em pré-operatório ou pós-operatório das clínicas de cirurgia vascular, cirurgia geral, neurocirurgia e cirurgia em proctologia, que consentiram em responder ao questionário da primeira etapa da pesquisa. O critério de exclusão foi ser menor de 18 anos.

A coleta de dados ocorreu em três etapas distintas denominadas: etapa I: Coleta de dados sobre fatores de risco de queda intrínsecos com um questionário desenvolvido a partir do Protocolo de 
Prevenção de Quedas do Ministério da Saúde (2013). Etapa II: Coleta de dados sobre incidente queda segundo o participante do estudo no momento da alta ou através da ligação telefônica após alta hospitalar. Etapa III: Coleta de dados sobre incidente queda segundo o livro de ocorrência do cenário de estudo e ficha de notificação de queda no setor de Qualidade e Educação Permanente do Hospital.

Na etapa I para a coleta de dados do declínio cognitivo, o entrevistador se baseou nas possíveis estratégias para identificá-lo, como perguntar "qual o ano? Onde estamos? Soletre a palavra mundo de trás para frente. Mostrar um relógio e uma caneta. Pergunte como chamam. Leia e execute a ordem: "feche os olhos".

Para identificar se o participante tinha ansiedade foram feitos dois questionamentos: "Você tem dificuldade de concentração?". O entrevistador também observava se existia comportamento de ansiedade do participante durante a entrevista.

Na depressão foram feitos algumas perguntas como: "Apresenta tristeza aparente? Apresenta tristeza relatada? Apresenta alteração do sono? Apresenta pensamentos suicidas? Apresenta pensamentos pessimistas?".

Para o fator de risco de queda "dificuldade de desenvolvimento das atividades de vida diária" no ambiente da enfermaria, o participante foi questionado quanto à realização das seguintes atividades sozinho: tomar banho, usar o banheiro, comer, vestir roupa, pentear o cabelo e andar.

A etapa I da pesquisa aconteceu no mês de janeiro de 2015, a etapa II nos meses de fevereiro e março de 2015 e a etapa III aconteceu de forma simultânea às etapas I e II.

Para análise das variáveis, utilizou-se o programa estatístico $\mathrm{R}^{\circledR}$ com a finalidade de computar os dados, realizar cálculos estatísticos de frequência absoluta e relativa.

O trabalho de mestrado em questão trata-se de uma pesquisa de campo envolvendo seres humanos, por isso foi submetido e aprovado pelo Comitê de Ética em Pesquisa (CEP), com o número de aprovação 850.633 em 30/10/2014.

\section{- RESULTADOS}

Ao total foram entrevistados 98 pacientes internados, no período de janeiro de 2015, durante os dias em que houve internação de pacientes, ou seja, de segunda a quinta-feira, total de 16 dias. Ocorreram neste período cinco $(5,1 \%)$ casos de queda.

Observa-se que neste estudo, $36(36,7 \%)$ dos entrevistados tinham idade maior que 65 anos. Entretanto, destacam-se quatro ( $80 \%$ ) casos de queda no qual os pacientes eram idosos.

Segundo a figura I, no grupo de pacientes que não caíram há distribuição simétrica (mediana de 61 anos) entre as faixas etárias, variando entre 28 e 87 anos, com média de 60 anos. No grupo dos pacientes que tiveram o desfecho queda a idade variou entre 45 e 84 anos, com média de 68 e mediana em 69 anos.

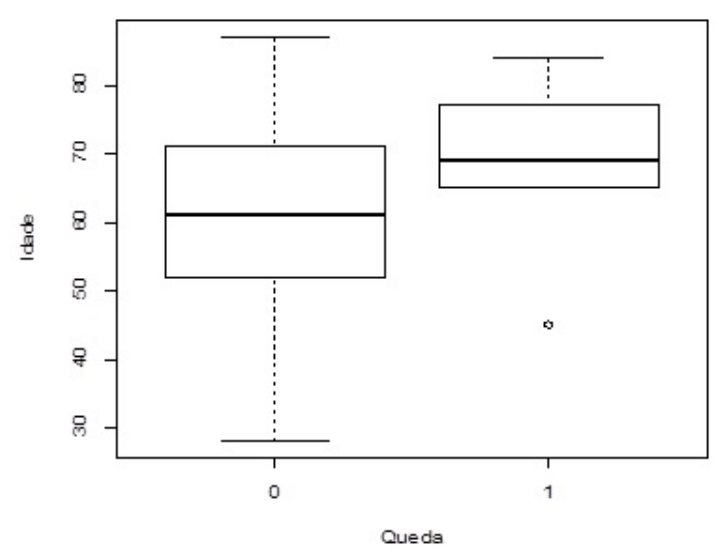

Figura 1 - Distribuição dos participantes que sofreram queda por idade ( $0=$ ausência do desfecho; $1=$ desfecho). Rio de Janeiro, RJ, Brasil, 2015 
Os fatores de risco para queda referentes à análise psicocognitiva do indivíduo encontrou-se para o declínio cognitivo um (1\%) paciente, para ansiedade 47 (48\%) e 26 (26,5\%) com depressão. Sobre o registro de queda, foram encontrados três $(60 \%)$ dos pacientes que caíram com quadro de ansiedade e dois (40\%) com depressão. Destacam-se dois pacientes que tinham depressão associada à ansiedade e sofreram queda.

Nesta pesquisa, a hipotensão postural apareceu como fator de risco para queda em $34(34,7 \%)$ dos participantes, a tontura em 35 (35,7\%), a anemia em 26 (26,5\%) e a hipoglicemia em $20(20,4 \%)$.

A hipotensão postural, a tontura e a hipoglicemia foram prevalentes em dois $(40 \%)$ dos pacientes que sofreram queda. Porém a anemia foi encontrada em quatro $(80 \%)$ dos entrevistados. Sobre a hipotensão postural e a tontura, observa-se que um dos pacientes que sofreu queda fazia uso de medicamento diurético e apresentava esses dois fatores de risco.

A presença de insônia foi descrita por 41 (41,8\%) dos entrevistados, apenas um apresentou queda. Do total de pacientes com incontinência ou urgência miccional, $21(21,4 \%)$ tinham este fator de risco, porém dois sofreram queda. O paciente que apresentava insônia com o desfecho queda também relatou incontinência ou urgência miccional.

Quando questionados sobre a dificuldade de desenvolvimento das atividades de vida diária no ambiente da enfermaria, ou seja, conseguir realizar atividades como: tomar banho, comer, vestir roupa, pentear o cabelo e andar, $36(36,7 \%)$ dos pacientes relataram ter dificuldade funcional para realizar alguma dessas atividades sem auxílio.

Sobre os pacientes com desfecho queda, três (60\%) não apresentavam dificuldade funcional e dois (40\%) tinham dificuldade.

Os problemas articulares foram descritos por $22(22,2 \%)$ dos pacientes do estudo. Destaca-se que um paciente que sofreu queda tinha esse fator de risco. Sobre a presença de deformidade nos membros inferiores, nove $(9,1 \%)$ pessoas tinham essa alteração física, sendo que um caso de queda tinha tal sintoma. Os dois pacientes com registros de queda citados estavam internados na clínica vascular.

O comprometimento sensorial do participante do estudo foi avaliado segundo dois critérios: a acuidade visual diminuída e a perda da audição. 73 (74,5\%) pacientes relataram algum tipo de comprometimento da visão, 16 (16,3\%) afirmaram ter dificuldade para ouvir.

O comprometimento da visão esteve presente nos cinco (100\%) pacientes com o desfecho queda, porém não foi descrito comprometimento da audição.

Observa-se que 31 (31,6\%) dos entrevistados afirmaram ter dificuldade para deambular na enfermaria. Em relação à amostra que sofreu queda, dois (40\%) pacientes apresentavam marcha alterada.

A polifarmácia (uso de quatro ou mais medicamentos) foi encontrada em 44 (45\%) dos participantes entrevistados. A associação no uso de alguns medicamentos, horários e vias de administração corroboram para o risco de queda.

Destaca-se que três $(60 \%)$ dos pacientes com desfecho queda faziam uso de mais de quatro medicamentos no dia da entrevista.

Referente ao grupo de pacientes que sofreram queda, observa-se que um paciente fazia uso de diurético, um usava digoxina e benzodiazepínico, outro fazia uso de insulina, diurético, antidepressivo e antipsicótico. Porém, dois pacientes com desfecho queda não faziam uso dos fármacos descritos no instrumento de coleta de dados.

O relato de queda prévia é um importante parâmetro a ser avaliado no momento da avaliação ao paciente. Foram descritos $29(29,6 \%)$ relatos de história de queda pelos entrevistados anterior à internação. Ao total, três $(60 \%)$ casos de quedas tinham história anterior do evento.

\section{- DISCUSSÃO}


Foram registrados cinco $(5,1 \%)$ de casos de queda neste estudo. Pesquisa desenvolvida em unidade de neurocirurgia, no período de sete meses, foram descritos oito $(8,2 \%)$ pacientes que apresentaram queda do total de 97 da amostra ${ }^{(2)}$. A média de queda em um Hospital Universitário durante 30 meses de avaliação das notificações em boletim de evento adverso, foi igual a 2,6 quedas no mês ${ }^{(5)}$.

Referente ao fator de risco idade maior que 65 anos, prevalente em $80 \%$ dos casos de queda. Sabese que as quedas, particularmente nas pessoas idosas, resultam de vários fatores que interagem. Os fatores mais significativos nos pacientes hospitalizados são: andar cambaleante, estado de confusão, incontinência ou a necessidade de usar o banheiro em muitas situações, queda anterior, o uso de sedativos e remédios para dormir. Por isso, as pessoas mais velhas são mais vulneráveis à queda ${ }^{(6)}$.

Pesquisa anterior desenvolvida por outro autor corrobora com os achados desta pesquisa, porque se notou maior prevalência de queda na faixa etária de 60 anos ou mais ${ }^{(5)}$. Em uma coorte prospectiva, $25 \%$ das quedas ocorreram em pacientes com idade superior a 65 anos internados na unidade de neurocirurgia e $\mathrm{UTI}^{(2)}$.

Referente à prevalência dos fatores de risco depressão e ansiedade, resultado similar foi encontrado em estudo com pacientes internados nas enfermarias de cirurgia geral em pré-operatório. Observouse $44,3 \%$ casos de ansiedade e $26,6 \%$ de casos de depressão. Referente ao paciente em pré-operatório, é frequente encontrar sintomas psicológicos de ansiedade e depressão que se confundem com sintomas da doença que originou a intervenção cirúrgica ${ }^{(7)}$.

Sobre o fator de risco hipotensão postural, prevalente em $34,7 \%$ dos participantes, é descrito na literatura científica que tal sintoma é comum nos idosos, tornando-os mais vulneráveis às quedas. $\mathrm{O}$ idoso é capaz de manter o controle postural, entretanto com a presença de doenças que interferem na regulação da pressão arterial aumenta-se a probabilidade de instabilidade postural ${ }^{(8)}$.

A presença de anemia foi encontrada em $80 \%$ dos casos de queda. Sabe-se que a diminuição da concentração de hemoglobina compromete o transporte de oxigênio para todos os tecidos, reduzindo o desempenho físico em pessoas anêmicas ${ }^{(9)}$.

Referente à presença de hipoglicemia como fator de risco para queda, as patologias crônicodegenerativas como a diabetes mellitus, são consideradas de longa duração e há a possibilidade de alterações agudas como a hiperglicemia ou hipoglicemia, que podem provocar quedas ${ }^{(10)}$.

A presença de insônia e/ou urgência urinária tornam o paciente em risco de queda devido à maior chance de tentar sair do leito durante o período noturno. O não reconhecimento do ambiente da enfermaria e do banheiro pelo paciente torna-o mais vulnerável ao incidente queda. $\mathrm{O}$ receio de acionar a equipe de enfermagem para auxiliar durante deslocamento até o banheiro expõe ainda mais o paciente ao risco de queda.

A insônia é um antecedente da ocorrência de queda, devido à sonolência posterior que ela provoca pela má qualidade do sono, gerando cansaço e movimentos perigosos. O paciente poderá no período noturno realizar caminhas errantes. Esta situação, quando ocorre em ambiente com iluminação insuficiente ou superfície irregular e escorregadia como o banheiro, torna o risco de queda evidente ${ }^{(11)}$.

A incontinência urinária foi objeto de estudo em um Hospital Universitário. A prevalência em adultos no cenário de pesquisa correspondeu a 35\%, sendo mais prevalentes na clínica cirúrgica, total de $48 \%$ da amostra. Destaca-se neste estudo o fato de $85,2 \%$ dos pacientes acordarem para urinar e $63 \%$ conseguem chegar ao banheiro a tempo ${ }^{(12)}$.

Observou-se que $60 \%$ dos casos de queda não apresentavam dificuldade funcional, o que contraria os dados encontrados na literatura que justificam essa relação, como estudo que mostrou que 133 $(66,5 \%)$ dos pacientes que sofreram queda durante período de internação hospitalar apresentavam incapacidade ou necessidade de ajuda para o autocuidado no momento da alta hospitalar ${ }^{(13)}$.

Setenta e seis dos participantes relataram não precisar de bengala ou muleta para locomoverse, porém $22(22,4 \%)$ precisavam destes objetos para auxílio à marcha. A queda aconteceu em dois $(40 \%)$ pacientes que faziam uso do dispositivo de auxílio, ambos eram idosos e tinham dificuldade no desenvolvimento das atividades de vida diária. 
O auxílio na deambulação poderá ser feito de outras formas, com auxílio pelo profissional de saúde, uso de andador e segurando-se no mobiliário e/ou parede. O uso de muleta/bengala/andador foi descrito em estudo do tipo coorte prospectivo em hospital de grande porte. Do total de pacientes que faziam uso destes dispositivos $(4,3 \%)$, quatro $(16,7 \%)$ sofreram queda durante internação ${ }^{(14)}$.

A presença de doença vascular é descrita como fator de risco para queda, assim como os problemas nos pés. A incidência de doença vascular foi igual a 15,8\% e o problema nos pés 10,5\% em estudo realizado com um grupo da terceira idade em um centro de saúde ${ }^{(15)}$.

A variável alteração nos pés foi descrita como fator de risco para queda em pacientes idosos acometidos por acidente vascular cerebral em associações de reabilitação. Foram consideradas as alterações como calos, unhas encravadas ou deformidades nos pé, com prevalência igual a 36,6\% da $\operatorname{amostra}^{(16)}$.

Sabe-se que a redução da acuidade visual pode levar à queda diretamente pela diminuição da estabilidade postural, ou indiretamente por reduzir a mobilidade e a função física ${ }^{(10)}$. Associação estatística entre o déficit visual e a ocorrência de queda foi descrita em pesquisa onde, dos pacientes que caíram, $88,6 \%$ possuíam este agravo ${ }^{(14)}$. Sendo assim, destaca-se o fator de risco comprometimento da visão encontrado no presente trabalho em $100 \%$ da amostra que sofreu queda como predisponente para o evento, portanto, devendo ser parâmetro importante avaliado durante a internação hospitalar, para o estabelecimento de medidas de prevenção.

Sabe-se que a diminuição da sensibilidade auditiva gera quadros de vertigem e dificulta o controle postural, principalmente ao realizar movimentos bruscos e mudança de direção, aumentando a ocorrência de queda ${ }^{(8)}$.

Estudo de coorte prospectivo em hospital de grande porte evidenciou que $10,4 \%$ dos pacientes que sofreram queda tinham marcha fraca e $25,4 \%$ marcha comprometida/cambaleante. Para o estabelecimento do risco elevado para quedas, a marcha comprometida/cambaleante foi um fator relevante ${ }^{(14)}$.

No paciente que sofreu queda, é relevante rever as medicações em uso, alterando ou suspendendo, sempre que possível, com objetivo de prevenir novos episódios de quedas. Recomenda-se particular atenção aos pacientes em uso de quatro ou mais fármacos e aqueles que utilizam os psicofármacos. Sendo notório como intervenção pelo enfermeiro monitorar o risco de queda no caso do paciente iniciar o uso do psicofármaco na internação, durante três dias, diariamente ${ }^{(17)}$.

O relato de história prévia de queda estava presente em $29,6 \%$ do total de entrevistados. Número similar foi descrito em estudo publicado que encontrou $21,6 \%$ de pessoas internadas com história anterior de queda ${ }^{(2)}$.

O Ministério da Saúde ${ }^{(1)}$ indicou os fatores predisponentes específicos que aumentam o risco de queda. Ao total são descritos 26 fatores. O foco principal é pôr em prática os ideais do Protocolo de Prevenção de Quedas do Ministério da Saúde ${ }^{(1)}$ e reduzir os casos de queda no cenário de estudo desta pesquisa. Portanto, pesquisa futura pretende aprofundar o assunto entorno do desenvolvimento de tecnologia educacional para reduzir a incidência de queda no cenário de estudo.

A construção e validação de instrumentos para identificação dos pacientes com risco de queda a partir da presença dos fatores é fundamental para nortear o trabalho da enfermagem na promoção de ações focadas na prevenção de queda ${ }^{(18)}$.

A construção da tecnologia educacional será modelo de capacitação para equipe de enfermagem da clínica cirúrgica visando os fatores de risco de queda mais prevalentes em relação à forma como ocorre o incidente neste cenário, com enfoque no perfil clínico do paciente, estratégias de prevenção pautadas na realidade estrutural, profissional e de acordo com os recursos disponíveis.

\section{CONSIDERAÇÕES FINAIS}

Sobre a prevalência dos fatores de risco de queda na amostra estudada, a média entre os pacientes que sofreu queda foi igual a nove, variando entre 17 fatores em um caso e dois em outro. A taxa de 
queda no mês de coleta de dados foi igual a 5,1\%. Porém ao desenvolver o estudo no cenário de coleta de dados, foi possível identificar falhas no processo de notificação do evento queda, pois nenhum caso de queda foi notificado da forma adequada, podendo, portanto, haver subnotificação do evento.

A prevalência dos fatores de risco intrínsecos se faz necessária com o intuito de estabelecer um perfil do paciente da clínica cirúrgica, mesmo não havendo escala institucional para avaliar o risco de queda no cenário do estudo. Salienta-se a importância de fazer avaliação correta do paciente no momento da internação e nos dias seguintes até a alta hospitalar, na tentativa de minimizar a ocorrência de quedas.

A associação dos fatores de risco no paciente que sofreu queda mostrou que $100 \%$ (cinco) dos pacientes tinham o fator de risco comprometimento da visão, $80 \%$ (quatro) apresentavam idade maior que 65 anos e anemia, $60 \%$ (três) apresentavam ansiedade, faziam uso de quatro ou mais medicamentos e tinham história prévia de queda.

A realização deste estudo irá contribuir com as publicações científicas na área, promovendo incentivo e auxiliando na construção de práticas de intervenção para outras Instituições de Saúde, contribuindo deste modo para o fortalecimento da prevenção de quedas nos diversos ambientes hospitalares do Brasil, e na descoberta dos reais fatores que levam os pacientes brasileiros a cair, pois grande parte das pesquisas desenvolvidas são de fora do país, o que representa realidades distintas.

A pesquisa desenvolvida apresentou como limitação a dificuldade de se obter o registro queda no cenário de estudo, devido o não uso da ficha de notificação padronizada para o evento adverso.

\section{REFERÊNCIAS}

1. Ministério da Saúde (BR), Agência Nacional de Vigilancia Sanitária (ANVISA) e Fundação Oswaldo Cruz (Fiocruz). Protocolo Prevenção de Quedas. PROQUALIS, 2013.

2. Diccini S, de Pinho PG, da Silva FO. Assessment of risk and incidence of falls in neurosurgical inpatients. Rev. Latino - Am. Enferm. 2008; 16(4): 752-7.

3. Severo IM, Almeida MA, Kuchenbecker R, Vieira DFVB, Weschenfelder ME, Pinto LRC, et al. Fatores de risco para quedas em pacientes adultos hospitalizados: revisão integrativa. Rev. esc. enferm. USP. 2014; 48(3): 540-54.

4. Teixeira TCA, Cassiani SHB. Análise de causa raiz de acidentes por quedas e erros de medicação em hospital. Acta paul. enferm. 2014; 27(2): 100-7.

5. Paiva MCMS, Paiva SAR, Berti HW, Campana AO. Caracterização das quedas de pacientes segundo notificação em boletim de eventos adversos. Rev. esc. enferm. USP. 2010; 44(1): 134-8.

6. National Patient Safety Agency. The third report from the patient safety observatory. Londres, 2007.

7. Marcolino JAMM, Suzuki FM, Alli LAC, Gozzani JL, Mathias LAST. Medida da ansiedade e da depressão em pacientes no pré-operatório. Estudo Comparativo. Rer. Bras. Anestesiol. 2007; 57(2): 157-66.

8. de Menezes RL, Bachion MM. Estudo da presença de fatores de riscos intrínsecos para quedas, em idosos institucionalizados. Ciênc. saúde coletiva. 2008; 13(4): 1209-18.

9. Fabian C, Olinto MTAO, Dias-da-Costa JS, Bairros F, Nácul LC. Prevalência de anemia e fatores associados em mulheres adultas residentes em São Leopoldo, Rio Grande do Sul, Brasil. Cad. Saúde Pública. 2007; 23(5): 1199205.

10. Costa ICP, Lopes MEL, de Andrade CG, Souto MC, da Costa KC, Zaccara AAL. Fatores de risco de quedas em idosos: produção científica em periódicos online no âmbito da saúde. Rev. Bras. Cienc. Saude. 2012; 16(3): 44552.

11. Vitor AF, Lopes MVO, de Araujo TL. Diagnóstico de enfermagem risco de quedas em pacientes com angina instável. Rev. Rene. 2010; 11(1): 105-13.

12. da Silva APM, Santos VLCG. Prevalência da incontinência urinária em adultos e idosos hospitalizados. Rev Esc Enferm USP. 2005; 39(1): 36-45. 
13. Marin HF, Bourie P,Safran C. Desenvolvimento de um sistema de alerta para prevenção de quedas em pacientes hospitalizados. Rev. latino-Am. Enfermagem. 2000; 8(3): 27-32.

14. Remor CP, Cruz CB, Urbanetto JS. Análise dos fatores de risco para queda de adultos nas primeiras 48 horas de hospitalização. Rev. Gaúcha Enfer. 2014; 35(4): 28-34.

15. Machado TR, Oliveira CJ, Costa FBC, Araujo TL. Avaliação da presença de risco para queda em idosos. Rev Elet Enf. 2009; 11(1): 32-8.

16. Costa AGS, de Araujo TL, Oliveira ARS, Morais HCC, da Silva VM, Lopes MVO. Fatores de risco para quedas em idosos. Rev Rene. 2013; 14(4): 821-8.

17. da Costa-Dias MJM, Oliveira AS, Moreira CN, Santos AS, Martins T, Araújo F. Quedas dos doentes internados em serviços hospitalares, associação com os grupos terapêuticos. Rev. Enf. Ref. 2013; 2(29): 105-114.

18. Vitor AF, Moura LA, Fernandes APNL, Botarelli FR, Araúdo JNM, Vitorino ICC. Risco de quedas em pacientes no período pós-operatório. Cogitare enferm. 2015; 20(1): 29-37. 